\title{
Tuberculosis cutánea. Reporte de un caso
}

\author{
Cutaneous tuberculosis. Case report
}

\author{
Dras. Elsa Bisero ${ }^{a}$, Graciela Luque $^{a}$, Karina Melillo ${ }^{a}$,María Inés Favier ${ }^{b}$, Alejandra Zapatac $y$ \\ María Soledad Cuello
}

\section{RESUMEN}

Se denomina tuberculosis cutánea a la enfermedad cutánea infecciosa crónica ocasionada por el Mycobacterium tuberculosis. Es poco frecuente y particularmente difícil de diagnosticar. Su incidencia, según la bibliografía, oscila entre el 1,5 y el $4 \%$ de todas las formas de tuberculosis extrapulmonares. Las formas clínicas dependen de la vía de llegada del bacilo a la piel, del estado inmunológico del paciente y del medio ambiente. Se presenta un caso de tuberculosis cutánea en un niño con lesiones dermatológicas crónicas, de evolución tórpida, sin respuesta a los tratamientos instituidos; la biopsia de piel mostró granulomas caseosos. Elobjetivoes describir un paciente con una presentación clínica infrecuente de esta enfermedad, destacar la importancia de su reconocimiento y tratamiento precoz, y evitar así la aparición de complicaciones y secuelas. Palabras clave: Mycobacterium tuberculosis, úlcera cutánea, tuberculosis cutánea.

\begin{abstract}
Cutaneous tuberculosis is a chronic infectious disease caused by Mycobacterium tuberculosis. It is not very frequent and particularly difficult to diagnose. It incidence ranges between 1.5 and $4 \%$ of all extrapulmonary tuberculosis, according to bibliography. The clinic presentations depend on the arrival via of the bacillus to the skin, the patient's immune state and the environment.

We show a cutaneous tuberculosis on a child with chronic dermatologic lesions, with torpid evolution, without response to treatments; the skin biopsy showed caseous granulomas. The aim is to show a patient with an infrequent clinic presentation of this disease, to emphasize the importance of an early recognition and treatment, avoiding the appearance of complications and sequels.
\end{abstract}

Key words: Mycobacterium tuberculosis, skin ulcer, cutaneous tuberculosis.

http:/ /dx.doi.org/10.5546/aap.2014.e93

a Servicio de Pediatría. Sección Neumonología Infantil.

b Servicio de Dermatología. Sección Dermatología Infantil.

c Servicio de Laboratorio. Sección Bacteriología.

d Servicio de Anatomía Patológica.

Hospital Nacional Prof. A. Posadas.

Departamento Materno-infantil. División Pediatría.

Correspondencia:

Dra. Elsa Bisero: elsibisero@yahoo.com.ar.

Conflicto de intereses: ninguno que declarar.

Recibido: 13-8-2013

Aceptado: 23-1-2014

\section{INTRODUCCIÓN}

En las últimas décadas, han aumentado las formas graves y extrapulmonares de tuberculosis, relacionadas con la inmigración, la epidemia del síndrome de inmunodeficiencia adquirida (SIDA), el aumento del número de personas institucionalizadas, el deterioro de las condiciones socioeconómicas y las carencias nutricionales, entre otras.

La tuberculosis cutánea es una enfermedad infecciosa crónica poco frecuente, particularmente difícil de diagnosticar. Su incidencia, según la bibliografía, oscila entre el 1,5 y el $4 \%$ de todas las formas de tuberculosis extrapulmonares. Aumenta en los pacientes infectados por el virus de inmunodeficiencia humana (VIH) y en países en desarrollo. ${ }^{1,2,3}$

El tegumento cutáneo es un órgano poco receptivo para el bacilo de Koch; esto condiciona manifestaciones menos frecuentes, más crónicas y con menos bacilos, a diferencia de lo observado en otras formas extrapulmonares.

Existe una gran variedad de formas clínicas que dependen de la vía de llegada del bacilo a la piel, del estado inmunológico del paciente y del medio ambiente.

Su diagnóstico se basa en los criterios absolutos y relativos de Robert J. Wilkinson, quien hace referencia al cultivo como criterio absoluto y a la historia clínica, examen físico, reacción tuberculínica, tuberculosis activa en otros órganos, bacteriología, histología de las muestras obtenidas y respuesta al tratamiento como criterios relativos.

\section{CASO CLÍNICO}

Niño de 8 años, procedente de la localidad de Moreno, provincia de Buenos Aires, de mal medio socioeconómico, con malas condiciones de higiene y hacinamiento, desnutrido. Tiene antecedentes de hipotiroidismo congénito (tratado con levotiroxina), episodios de obstrucción bronquial recurrente desde los dos años, otitis y laringitis a repetición e hipoacusia. Antecedentes familiares de asma.

Consulta a Dermatología por presentar lesión alopécica ulcerada en el cuero cabelludo 
de más de dos meses de evolución, máculas hipopigmentadas rugosas al tacto en las mejillas, lesión en la región suprapúbica redondeada con pérdida de sustancia, ulcerada y dolorosa, y blefaritis en el ojo izquierdo (Figuras 1, 2 y 3).

Los diagnósticos presuntivos dermatológicos fueron micosis profundas, epiteliomas, sífilis y leishmaniasis.

Auxiliares diagnósticos: glóbulos blancos, $6600 / \mathrm{mm}^{3}$; hemoglobina, 12,6 g/dl; plaquetas, $279000 / \mathrm{mm}^{3}$. Función renal y hepática normal.

FIgURA 1. Lesión ocular al momento del ingreso

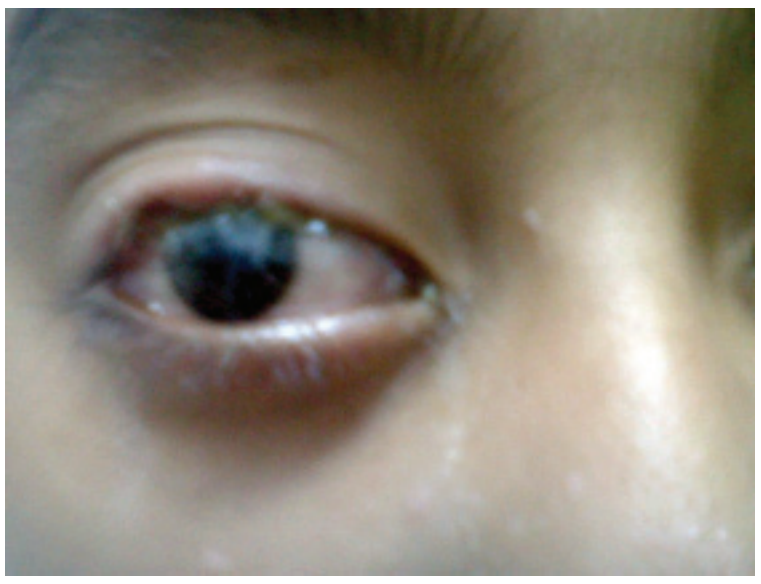

Secreción purulenta de párpado superior, caída de pestañas, ojo rojo y chalazión.

FIGURA 2. Lesión en el cuero cabelludo

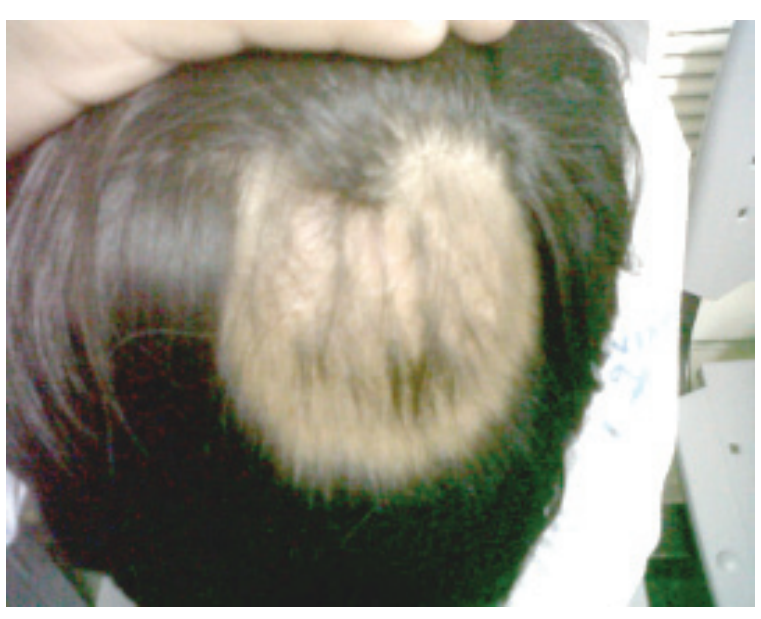

Lesión alopécica ulcerada en el cuero cabelludo.
Perfil lipídico normal. Hormonas tiroideas normales. Serologías: VDRL y HIV negativos. Proteinograma con inmunoelectroforesis normal. Inmunoglobulinas ( $\mathrm{IgG}$ ) normales. Hisopado nasal negativo. Orina normal. Examen micológico de las lesiones en cara y cuero cabelludo con resultado negativo. Serologías para Histoplasma capsulatum: IgM e IgG negativas. Se solicita una consulta oftalmológica, en la que se informa secreción purulenta de párpado superior, caída de pestañas, ojo rojo y chalazión.

Se indicó griseofulvina y se incorporó cefalexina por sospecha de sobreinfección bacteriana.

A las dos semanas de tratamiento, se observa un empeoramiento de las lesiones en el cuero cabelludo y la región suprapúbica (aumento de tamaño y mayor secreción).

Se programa una biopsia de las lesiones del cuero cabelludo, la región suprapúbica y la cara.

Debido a la mala evolución, se realizauna consulta con Neumonología Infantil, en la que, por la cronicidad del proceso, la falta de respuesta a los tratamientos instituidos y el tipo de lesiones, se plantea una probable tuberculosis cutánea.

Presentaba vacunación BCG con cicatriz en la etapa neonatal.

Se solicita una prueba tuberculínica (2 U PPD-RT23) al momento del ingreso y a los dos meses de tratamiento, que fue de $0 \mathrm{~mm}$ en ambas oportunidades. Radiografía y tomografía de tórax de alta resolución normales. Ecografía abdominal normal. Radiografía de ambas caderas y cráneo normales. Foco de contagio tuberculoso: negativo.

FIgURA 3. Lesión suprapúbica al momento del ingreso

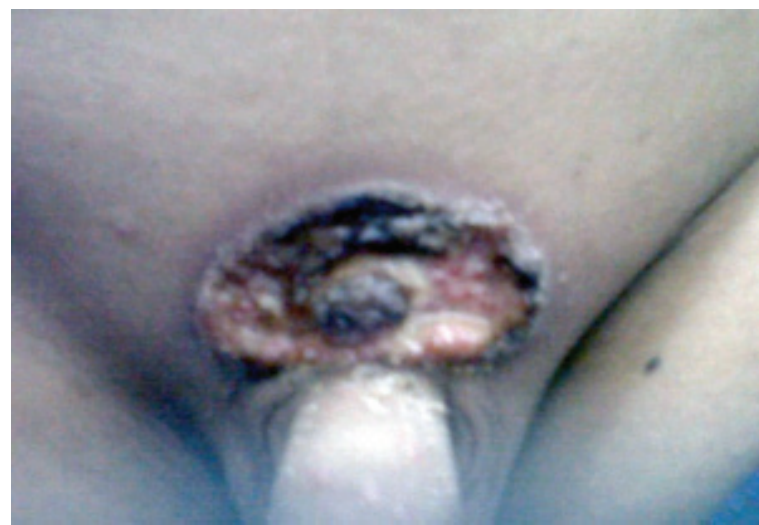

Lesión en región suprapúbica redondeada con pérdida de sustancia, ulcerada y dolorosa. 
Se recibe anatomía patológica de la lesión suprapúbica: dermatitis granulomatosa con sectores de necrosis caseosa. Ziehl-Nielsen (ZN) y ácido periódico de Shiff (PAS) negativos. Los cultivos a los 30 y 45 días fueron negativos. Se confirma el diagnóstico de tuberculosis cutánea, forma gomosa; se inicia un tratamiento con isoniacida $(\mathrm{H})(5 \mathrm{mg} / \mathrm{kg} / \mathrm{d})$, rifampicina $(\mathrm{R})$ (10 mg/kg/d), pirazinamida (Z) $(25 \mathrm{mg} / \mathrm{kg} / \mathrm{d})$, etambutol $(\mathrm{E})(20 \mathrm{mg} / \mathrm{kg} / \mathrm{d})$ y vitamina $\mathrm{B}_{6^{\prime}}$, con buena evolución de las lesiones a los dos meses (Figura 4).

\section{COMENTARIO}

La tuberculosis cutánea es causada, en la mayoría de los casos, por Mycobacterium tuberculosis y, raramente, por Mycobacterium bovis.

La vía de infección puede ser exógena o endógena (por contigüidad, hemática o linfática). ${ }^{4}$

M. tuberculosis ingresa al tejido cutáneo a través de abrasiones o heridas menores, colocación de pendientes, inyecciones con jeringas no esterilizadas, accidentes durante las necropsias, circuncisiones rituales, extracciones dentales, tatuajes, vacunación BCG. Se reporta, en raras ocasiones, por contacto sexual con pacientes que tienen tuberculosis genitourinaria.

Las reacciones en la piel dependen de varios factores, que incluyen el grado de inmunidad, la virulencia del bacilo y el tamaño del inóculo. ${ }^{5}$

El diagnóstico es difícil y en general tardío, debido a las distintas presentaciones con signos y síntomas inespecíficos que obligan a investigar otras etiologías, tal como ocurrió en nuestro paciente. ${ }^{6}$

El escrofuloderma y el lupus vulgar son las formas de tuberculosis cutánea más frecuentes., ${ }^{2,7}$

FIgURA 4. Lesión suprapúbica. Evolución a los dos meses de tratamiento

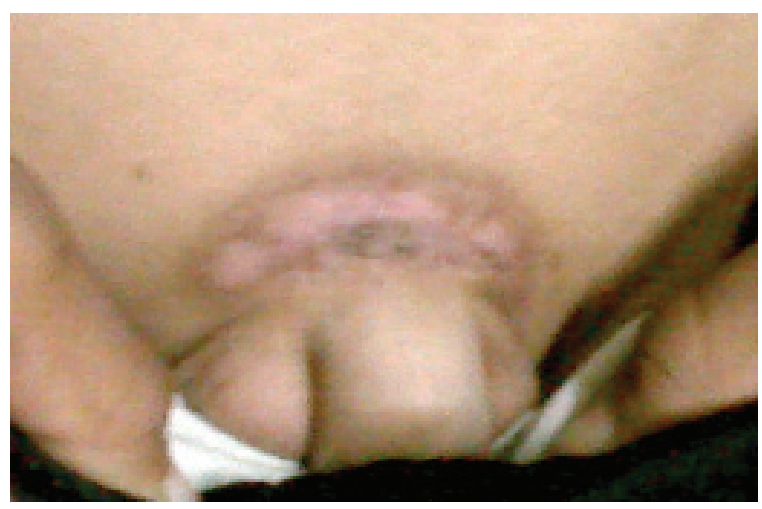

No existe consenso en cuanto a una clasificación. Beyt y col., categorizan las lesiones según la vía de infección y excluyen las formas de presentación atípicas y las tubercúlides. Tappeiner y col., amplían la clasificación de Beyt incluyendo las formas causadas por la vacunación BCG y las tubercúlides. ${ }^{8,9}$

La clasificación de Sehgal y col., toma en cuenta al agente infeccioso, la inmunidad de huésped y la ruta de infección. ${ }^{10,11}$

Las formas ulcerosas presentan un crecimiento excéntrico progresivo sin tendencia a la curación; son de bordes definidos, tal como lo observado en la lesión suprapúbica de nuestro paciente. Se planteó el diagnóstico diferencial con micosis profundas, epiteliomas, sífilis y leishmaniasis. Nuestro paciente presentó VDRL negativa; serología para Histoplasma capsulatum: inmunoglobulinas M y G negativas; y biopsia de piel con Giemsa y cultivo negativo para micosis.

El goma tuberculoso requiere diagnósticos diferenciales con gomas micóticos y sifilíticos, tumores benignos y malignos.

Otros diagnósticos diferenciales incluyen tularemia, fiebre por arañazo de gato, esporotricosis y otras enfermedades ulcerogranulomatosas.

La radiografía de tórax fue normal, al igual que la tomografía de tórax de alta resolución. La bibliografía reporta afectación pulmonar en alrededor del $20 \%$ de los casos. ${ }^{12}$

La sensibilidad tuberculínica es menor que en otras formas clínicas de tuberculosis; pueden ser negativas en pacientes gravemente enfermos, como el caso presentado. ${ }^{13}$

Por anamnesis, evolución tórpida de las lesiones, sin respuesta a los tratamientos instituidos, estudios complementarios que descartaron otras etiologías y la biopsia de piel con patrón granulomatoso y necrosis caseosa, se asume que se trataría de una tuberculosis cutánea gomosa múltiple de distribución asimétrica, que es una variante de la tuberculosis colicuativa. Su presentación es rara, se desarrolla a partir de un foco oculto y es más frecuente en pacientes desnutridos e inmunosuprimidos como nuestro paciente. Se produce por diseminación hematógena de la micobacteria. Clínicamente, se caracteriza por abscesos subcutáneos fluctuantes, no dolorosos, únicos o en sitios múltiples. La localización más frecuente se registra en las extremidades, tronco y cabeza.

Como informan la mayoría de los autores, el cultivo fue negativo. ${ }^{14}$

El manejo de la tuberculosis cutánea corresponde al de las tuberculosis extrapulmonares. ${ }^{4}$ 
Algunos autores mencionan la cirugía para acelerar la curación, realizan drenajes y desbridamientos quirúrgicos en el escrofuloderma y el goma.

La criocirugía o extirpación simple está reservada para la tuberculosis verrugosa. Nuestro paciente no requirió ningún procedimiento quirúrgico. ${ }^{15}$

El pronóstico en sí es favorable; depende del curso que tome la afección en el organismo, de la edad del paciente, del estado inmunológico y de la virulencia del $M$. tuberculosis. Actualmente, el niño concurre a controles periódicos, con buena evolución clínica, ganancia de peso, franca mejoría de las lesiones a los dos meses de tratamiento y laboratorio dentro de parámetros normales.

\section{CONCLUSIÓN}

La tuberculosis cutánea es infrecuente. Suele pasar desapercibida por mucho tiempo. $\mathrm{Su}$ diagnóstico debe ser considerado tanto en pacientes inmunocompetentes como en inmunodeprimidos que presenten lesiones crónicas en la piel, sin respuesta a los tratamientos instituidos.

\section{Agradecimiento}

Dra. Graciela Pizzariello por su constante dedicación a los pacientes, calidad humana y vocación docente.

\section{BIBLIOGRAFÍA}

1. González Montaner LJ,González Montaner P. Tuberculosis cutánea. En: Tuberculosis 2010;11:299-302. [Consulta: 4 de abril de 2014]. [Disponible en: www.bgb.biogen.com/ tuberculosis/pagina_299.php].
2. Pizzariello G, Fernández Pardal P, D’atri G, Novac V, et al. Espectro clínico de Tuberculosis cutánea. Rev Argent Dermatol 2008;89:177-87.

3. Singal A, Sonthalia S. Cutaneus tuberculosis in children: the Indian perspective. Indian J Dermatol Venereol Leprol 2010;6:494-503.

4. Comité Nacional de Neumonología, Comité Nacional de Infectología, Sociedad Argentina de Pediatría. Criterios de diagnóstico y tratamiento de la tuberculosis infantil. Arch Argent Pediatr 2002;100(2):159:78.

5. Calderón M, Rufasto M, Cáceres $H$, Torres $R$, et al. Tuberculosis cutánea verrugosa simulando cromomicosis en un niño. A propósito de un caso. Dermatol Pediatr Lat 2003;1:52-5.

6. SavinJA, Wilkinson DS. Mycobacterial Infections including Tuberculosis. En: Roo A, Wilkinson DS, Ebling FJG, et al. (eds.). Texbook of Dermatology. London: BlackwellScientific, 1986;48(1):791-822.

7. Concha RM, Fich SF, Rabagliati B, Pintos SC, et al. Tuberculosis Cutánea: Reporte de 2 casos y revisión de la literatura. Rev Chil Infect 2011;28(3):262-8.

8. Beyt BE, Ortbals DW, Santa Cruz DJ, Kobayashi GS, et al. Cutaneous mycobacteriosis: analysis of 34 cases with a new classification of the disease. Baltimore. Medicine 1980;60:95109.

9. Tappeiner G, Wolff K. Tuberculosis and other mycobacterial infections. En: Freedberg IM, Eisen AZ, Wolff K, Austen KF, et al., (eds.). Fitzpatrick's Dermatology in General Medicine. 6th ed. EE. UU.: Mc Graw-Hill; 2003.Págs.1933-49.

10. Sehgal VN. Cutaneous tuberculosis. Dermatol Clin 1994;12:645-53.

11. Panzarelli A. Tuberculosis Cutánea. Derm Venez 1998;36:4551.

12. Vashisht P, Sahoo B, Kharana N, Reedy BS. Cutaneus tuberculosis in children and adolescents: A cliicohistological study. J Eur Acad dermatol venerol 2007;21:40-7.

13. Tincopa Wong OW, Sánchez Saldaña L. Tuberculosis cutánea. Artículos de revisión. Dermatol Peru 2003;13(3): 195-214.

14. Tincopa O, Jave-Morales B. Tuberculosis Cutánea: estudio clínico, histopatológico y de frecuencia. Dermatol Peru 1999;9:10-8.

15. Rodríguez Peralto JL, Saiz A, Segurado A. Tuberculosis cutánea. En: Herrera Ceballos E, Moreno Carazo A, Requena Caballero L, Rodríguez PeraltoJL. Dermatología: Correlación Clínico-Patológica. Barcelona: Área Científica Menarini; 2007;597-600. 